\title{
Effect of Reheating to the Semisolid State on the Microstructure of the A356 Aluminum Alloy Produced by Ultrasonic Melt-Treatment
}

\author{
W. Khalifa ${ }^{1,2, a}$, Y. Tsunekawa ${ }^{1, b}$ and M. Okumiya ${ }^{1, c}$ \\ ${ }^{1}$ Toyota Technological Institute, 2-12-1, Hisakata, Tempaku, Nagoya, 468-8511, Japan \\ ${ }^{2}$ Cairo University, Dept. Mining, Petroleum and Metallurgical Engineering, 12613, Giza, Egypt \\ awkhalifa@toyota-ti.ac.jp, btsunekawa@toyota-ti.ac.jp, 'okumiya@ toyota-ti.ac.jp
}

\begin{abstract}
Keywords: reheating/soaking, semi-solid, non-dendritic, microstructure evolution, ultrasonic melt
\end{abstract} treatment, Al-Si alloys, entrapped eutectic, growth rate.

\begin{abstract}
The effect of reheating to the semisolid state (soaking treatment) on the microstructure evolution of the A356 aluminum alloy prepared by ultrasonic melt treatment was studied in this paper. The results showed that in general the longer the soaking process the larger and the more round the grains obtained. Higher roundness occurs at shorter soaking times in the fine-grained ascast samples, and at longer times in the inhomogeneous or the coarser-grained as-cast structures. The optimum thixotropic condition (high roundness, 0.72 , and small globule sizes $<90 \mu \mathrm{m}$ ) are achieved after $5 \mathrm{~min}$. soaking in the samples treated by UST at 623 and $620^{\circ} \mathrm{C}$, which is the typical soaking time dictated by the industrial practice in SSM. The amount of entrapped eutectic as observed after soaking treatments is uniquely very small, suggesting that the UST-treated ingots will have better formability in the semisolid state. The growth rate constants are substantially low: in the order of $479-748 \mu \mathrm{m}^{3} / \mathrm{s}$. These growth rate constants are much lower than those reported for MHDcast A356 ingots. The growth rates of the samples produced by UST in the liquid state (i.e., 626, 623 and $620^{\circ} \mathrm{C}$. Note that liquidus temperature is $619^{\circ} \mathrm{C}$ ) are lower than those of the samples treated in the semi-solid temperatures, i.e., 617 and $614^{\circ} \mathrm{C}$. The Ostwald ripening is most likely the dominant growth mechanism in the UST-treated samples during the soaking treatments. These results reveal the feasibility and competence of UST as a potential route for thixotropic feedstock production.
\end{abstract}

\section{Introduction}

The semisolid metal processing (SSM) provides high integrity with near to net shape products and close dimensional control even in complex parts. It is based on casting or forming semisolid slurry of non-dendritic (typically globular) solid particles dispersed in a metallic liquid matrix. The unique rheological properties of the metal semisolid slurry originate from the non-dendritic nature of the microstructure. The current SSM technologies can be divided into thixoforming / thixocasting and rheocasting processes. The former processes use pre-cast billet feedstock with non-dendritic structure, while the latter ones convert the liquid metal directly into slurry for casting. Due to the true high integrity and improved properties, the SSM finds wide applications in automotive, aerospace and other critical industries. The billets for thixoforming processes can be prepared by several techniques such as mechanical stirring, magnetohydrodynamic (MHD) stirring, stress induced and melt activated (SIMA) process, spray casting, liquidus casting, chemical grain refining, and ultrasonic treatment (UST) [1]. The MHD stirring is the most widespread practice for feed stock production. The main disadvantages of this technique are the high production cost (up to $50 \%$ of the component cost), microstructure non-uniformity in the cross section of the cast billet, and nonspherical particle morphology. The other techniques have limited commercial application due to technological drawbacks, high cost of production and in some cases limited applicability to certain alloys [1]. Thus, there is a definite need for economic and feasible SSM process. 
Ultrasonic treatment of molten metals, on the other hand, can effectively produce metal slurry with fine, non-dendritic and homogeneous microstructure suitable for SSM processing. The technology is not complex and offers other advantageous such as degassing, enhanced grain refinement effects [2], and energy saving [3]. In addition, the effective treatment time can be considerably short: ultrasonic treatment of $15 \mathrm{~s}$ at $1-10^{\circ} \mathrm{C}$ above the liquidus temperature provides fine non-dendritic structure [4]. In this paper we attempt to characterize the response of the USTtreated A356 alloys to the isothermal soaking treatments to determine the feasibility of the UST technology as an alternative route for thixotropic feedstock production.

\section{Experimental Procedure}

Ingots the A356 alloy, grain-refined with $\mathrm{Ti}$, were cut into pieces weighing 230 to $245 \mathrm{~g}$ and melted in alumina crucibles using resistance furnace. At about $650-660^{\circ} \mathrm{C}$, the crucible is transferred to the ultrasonic treatment (UST) setup [4]. The ultrasonic horn and the treatment furnace are kept at the same temperature range of the melt. The furnace temperature is adjusted so that the system cools down to the specified treatment temperatures, namely: $626,623,620,617,614,611$, and $608^{\circ} \mathrm{C}$ (Note that the liquidus temperature is $619^{\circ} \mathrm{C}$ ), then ultrasonic treatments are conducted by the top transmission method for 15 seconds. The melt is cast directly into a steel cup-mould kept at $100^{\circ} \mathrm{C}$.

The ingots (70mm-diameter) were then cut into two halves; one of them was cut further into 6 pieces, with cuts done from the perimeter to the center of the ingot. The pieces of each sample were separately reheated to the semisolid state using a salt bath of $50 \%$ sodium nitrate and $50 \%$ potassium nitrate kept at about $586 \pm 2{ }^{\circ} \mathrm{C}$ and soaked at this temperature for $1,5,15,30$ and 60 minutes, and quenched in water after soaking. The bath temperature went down to about $565-570^{\circ} \mathrm{C}$ upon sample immersion and heats up to the soaking temperature in 1-2 minutes. After restoring the bath temperature, the soaking time is counted. Samples of the base alloy conventionally cast into graphite and metallic moulds (No UST-G and No UST-M) were reheated in the same manner.

The flat surface (i.e., half of the ingot cross-section) of each sample was polished and etched with Keller's reagent. Image analysis was carried out to study the effect of reheating treatment on the microstructure of the ultrasonically treated samples. The equivalent grain size, roundness, and aspect ratio were investigated in each sample. The equivalent grain size (globule size) is defined as the diameter of a circle with the same cross-section as the grain in section. The roundness which is also called the sphericity, is defined as $4 \pi \mathrm{A} / \mathrm{P}^{2}$, (where $\mathrm{A}$ and $\mathrm{P}$ are the area and perimeter of the grain/globule, respectively). Several workers use this property in the form of the shape factor which is ( $1 /$ sphericity). The aspect ratio which is also called elongation factor is the ratio of maximum and minimum lengths of the rectangle with smallest area that can be drawn around the grain. About 15 and 10 fields (each field is about $4696527 \mu^{2}$ ) were analyzed in the inner and outer zones of the sample, respectively, with a total of 986 fields analyzed. The outer zones extend to about $2.5 \mathrm{~mm}$ from the surface. A filter for the smallest size of grains to be analyzed was used here to avoid considering the residual eutectic aluminum in the analysis. This may exclude some small $\alpha-\mathrm{Al}$ globules and thus, the average grain areas and sizes reported may be little larger than their real values. The total number of aluminum grains considered in the quantitative analysis was about 343,189 grains over 51 treatment conditions.

\section{Results and Discussion}

The results of the quantitative image analysis showed that generally the longer the soaking time the larger and the more round the grains obtained. The diagrams of Fig. 1 show this behavior clearly. Fig. 1(a) shows that the average aluminum grain/globule sizes increase with the soaking time. The roundness (Fig. 1(b)) also increases continuously until it reaches the highest value at the longest soaking time. This behavior was observed for all the starting materials regardless of the processing conditions before soaking. The best microstructures obtained in terms of size, roundness and 
homogeneity of grain structure over the cast piece regions were observed in the samples prepared by ultrasonic treatment at 620,623 and $626^{\circ} \mathrm{C}$, where globular grains of small sizes were seen to be dominant in the microstructure. The samples treated by ultrasonic vibration at $617^{\circ} \mathrm{C}$ and lower, responded differently to the reheating process where fine dendritic grains become globular easily after short reheating times, while the large dendritic grains dispersed among the finer grains of the as-cast structure, exhibited slow response producing inhomogeneous grain structure even after long reheating treatments (30 and 60 minutes). Among the different UST-treatment conditions, almost all the minimum grain sizes and the highest roundness values were seen in the samples treated at $623^{\circ} \mathrm{C}$.

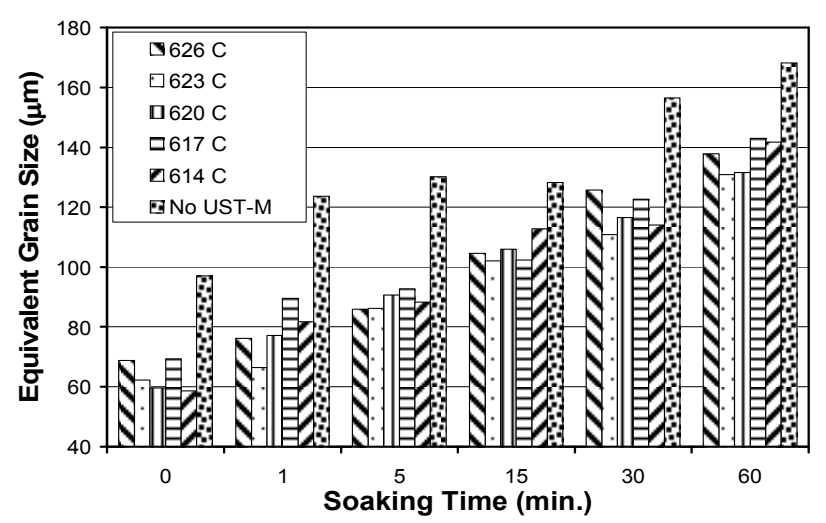

(a)

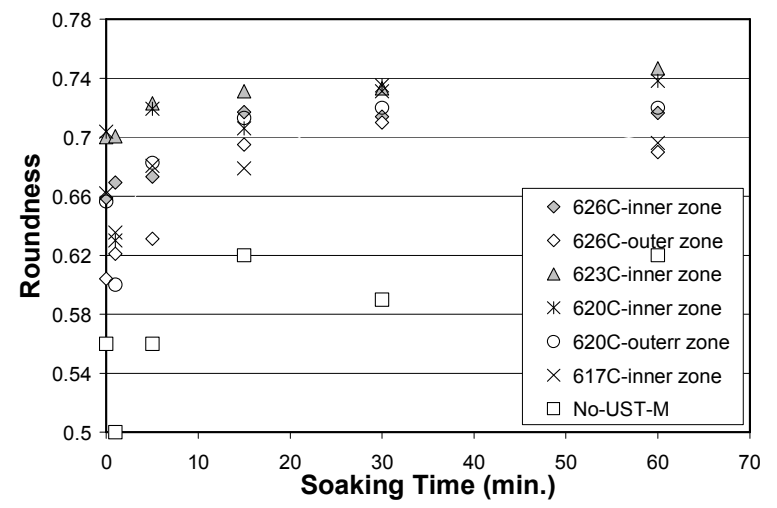

(b)

Fig. 1 Effect of soaking time on the (a) equivalent grain size, and (b) roundness of the $\alpha$-aluminum phase in UST-treated samples at different temperatures.

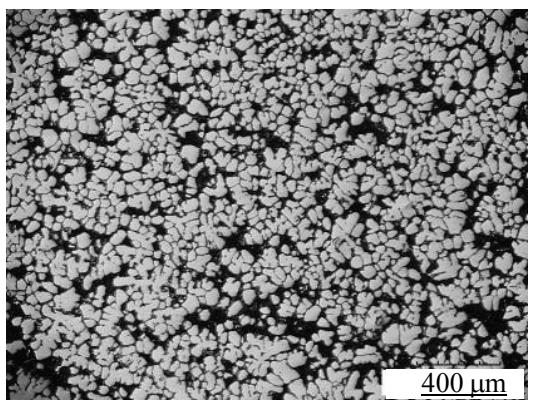

(a)

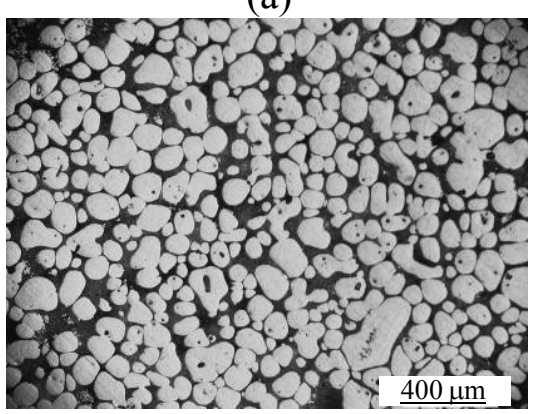

(d)

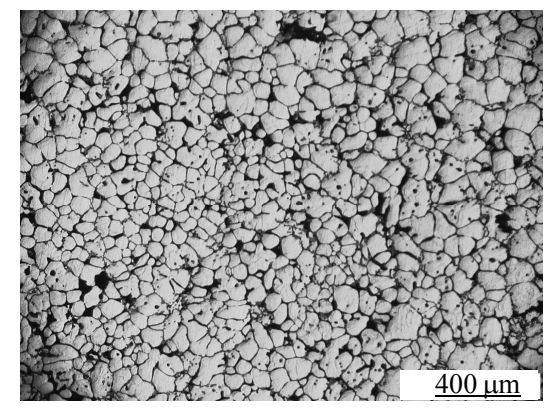

(b)

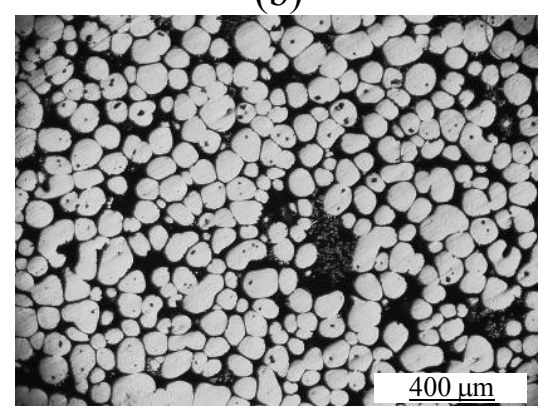

(e)

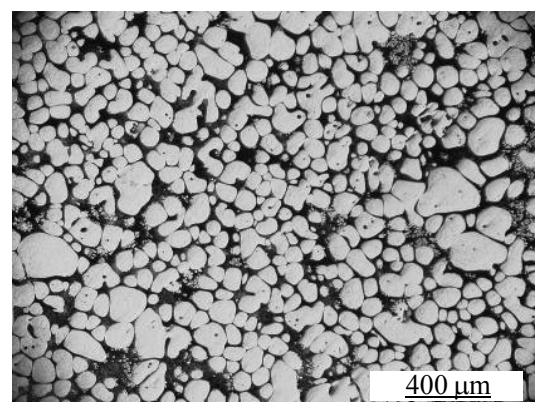

(c)

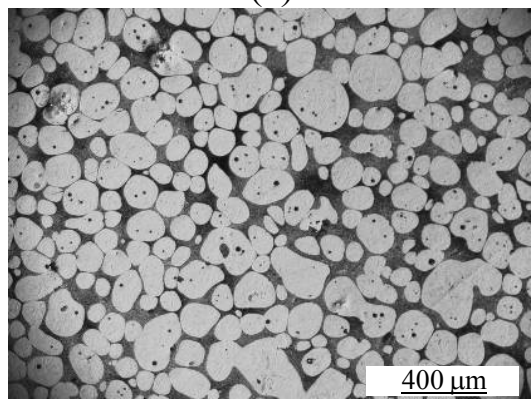

(f)

Fig. 2 The effect of soaking time on the microstructure of the sample treated by ultrasonic vibrations at $623^{\circ} \mathrm{C}$ : (a) as-cast, (b) $1 \mathrm{~min}$. (c) $5 \mathrm{~min}$., (d) $15 \mathrm{~min}$., (e) $30 \mathrm{~min}$., and (f) $60 \mathrm{~min}$.

As can be seen from Fig. 2, the aluminum grains in the inner zone of the casting become more globular after soaking, indicating the good response of the UST-treated alloys to the soaking treatment. The ingot's outer zones containing fine dendritic grains and rosettes in the as-cast condition also exhibited similar changes in morphology until they become globular at longer soaking treatments. High roundness $(>0.7)$ occurs at shorter soaking times in the fine-grained ascast samples, and at longer times in the inhomogeneous or the coarser-grained as-cast structures (see 
Fig. 1(b)), which is in agreement with the behavior of samples produced by electromagnetic stirring [5]. It is worth noting here that high roundness occurs after 5 minutes soaking in the samples treated by UST at 623 and $620^{\circ} \mathrm{C}$ (Fig. 2 and Fig. 3), indicating that the UST-treated samples ripen to an optimum thixotropic condition (i.e., high roundness, 0.72 , and small globule sizes, $<90 \mu \mathrm{m}$ ) at the typical soaking time dictated by the industrial practice in SSM (i.e., 5 minutes) [6].

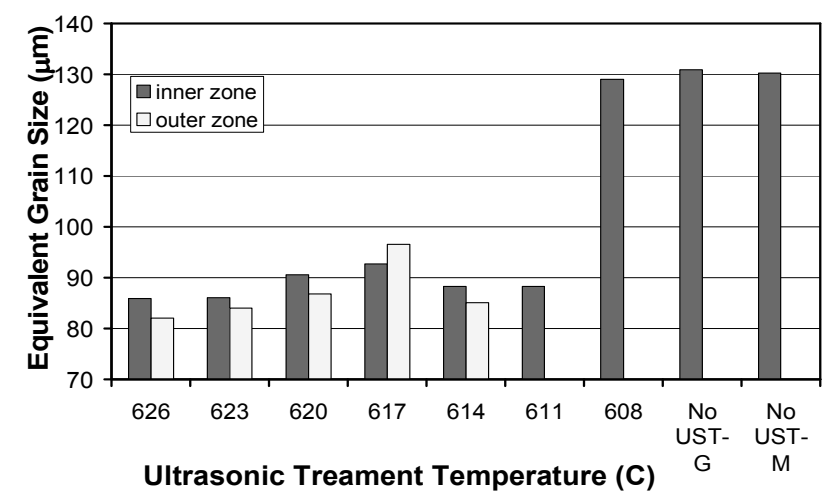

(a)

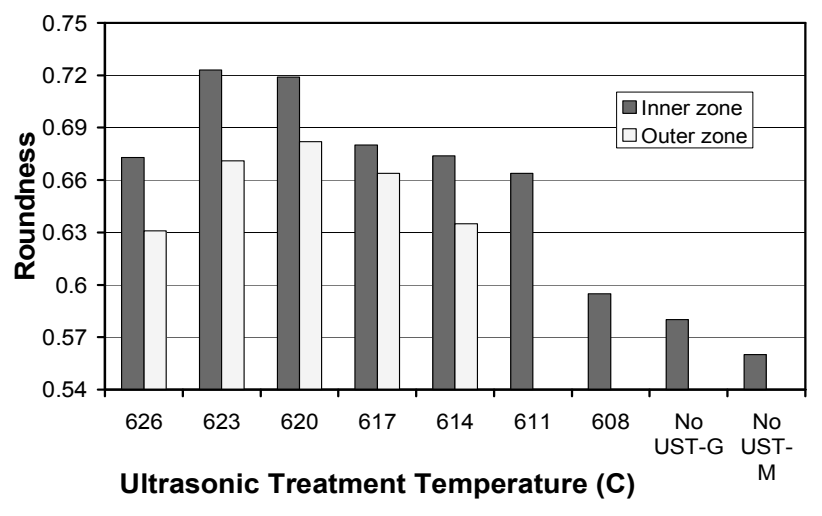

(b)

Fig. 3 Effect of soaking for 5 minutes on the (a) equivalent grain size, and (b) roundness of the $\alpha-\mathrm{Al}$ phase in samples treated by ultrasonic vibrations for $15 \mathrm{~s}$ at different temperatures.

It is also clear from the micrographs of Fig. 2 that the amount of entrapped eutectic is very small. The same observation holds for the samples ultrasonically treated at the liquid and semisolid states down to $614^{\circ} \mathrm{C}$. In comparison with microstructures reported by other researchers for samples produced by MHD, spray casting, SIMA [6,7], it is clear that the UST-treated samples are uniquely characterized by very low amount of entrapped eutectic. This suggests that the ultrasonically treated alloys will have better formability in the semisolid state since more liquid will be available between globules facilitating their flow during die filling. On the other hand, solid bridging in the die thin sections will be minimized since the globule sizes are smaller in this case. One more advantage is expected during solidification: the entrapped liquid creates internal stresses due to the shrinkage mismatch with the surrounding $\alpha$-solid which eventually can cause a gap with the surrounding $\alpha$-solid if the interfacial bond fails [8], detracting form the casting integrity. Little entrapped liquid is

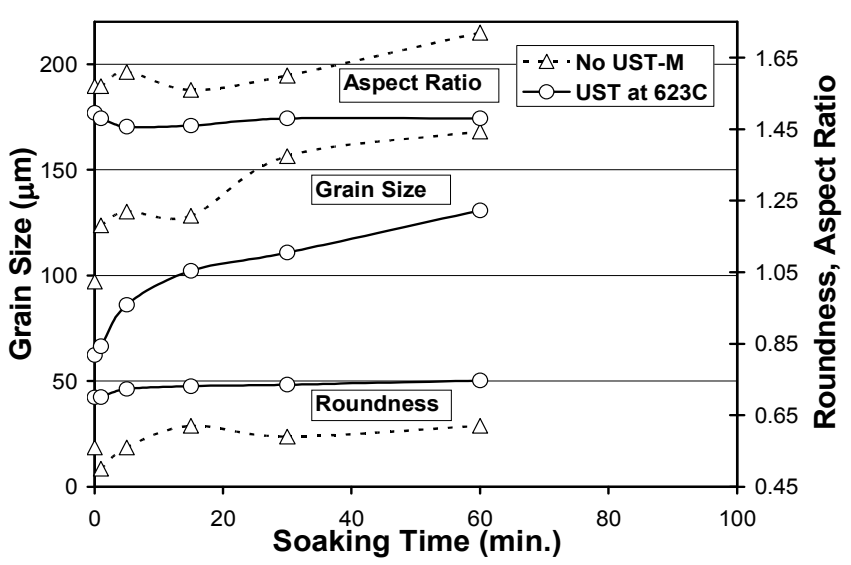

Fig. 4 Effect of soaking time on the grain size, roundness and aspect ratio of the UST-treated and non-treated samples. advantageous in this respect.

The size and morphology of the aluminum grains after reheating depend mainly on the original as-cast structure which was controlled to different extents by the ultrasonic treatment, and the time elapsed in the semi-solid state. As can be seen from the Fig. 4, the changes in size and morphology of the aluminum grains depend mainly on the as-cast structure. Although grain sizes increase continuously with soaking time, the difference between the two groups of ultrasonically-treated at $623^{\circ} \mathrm{C}$ (UST at $623^{\circ} \mathrm{C}$ ) and that of no ultrasonic treatment (No UST) continues so that the grain sizes of the former case remain smaller than the latter at all soaking times examined. The same behavior was also seen in the morphology of grain where the roundness and aspect ratio of the ultrasonically-treated samples remain much better than those of the non-UST-treated samples. 
During the soaking/ripening treatment, the $\alpha$-solid globules grow in size and acquire more curved surfaces with a gradual increase in roundness called globularization. The driving force for globularization is the thermodynamic tendency of minimizing the surface energy of the $\alpha$-solid. The growth mechanisms proposed for the ripening in the semi-solid state are the grain coalescence and the Ostwald ripening [5,6]. The grain coalescence takes place if two or more neighboring grains have low angle solid-solid grain boundaries or if the grains form coherent twins within the solid skeleton in the semi-solid state. The probability of having such neighboring grains in Al-7wt.\% Si is $7 \%$. Based on these data, the formation of agglomerates by low-energy grain boundary coalescence is seen less probable [6]. The grain coalescence mechanism is usually used to rationalize the rapid or unexpected grain growth during the soaking experiments. The globularization of the UST-treated samples is discussed below.

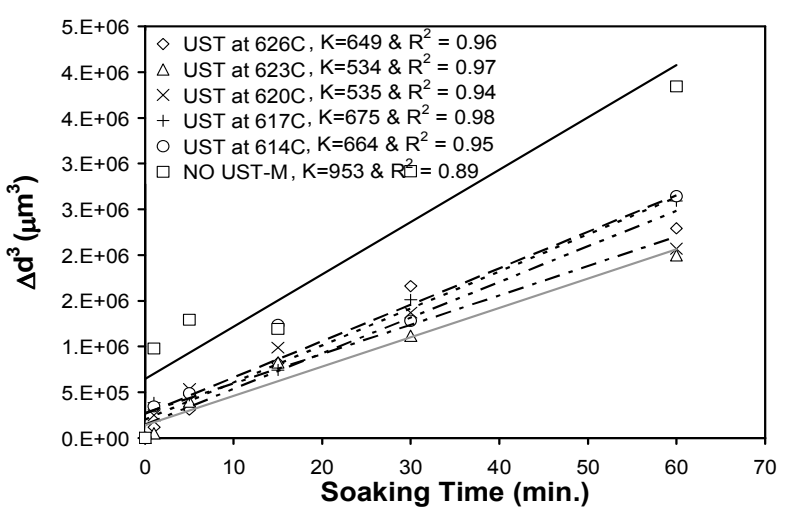

(a)

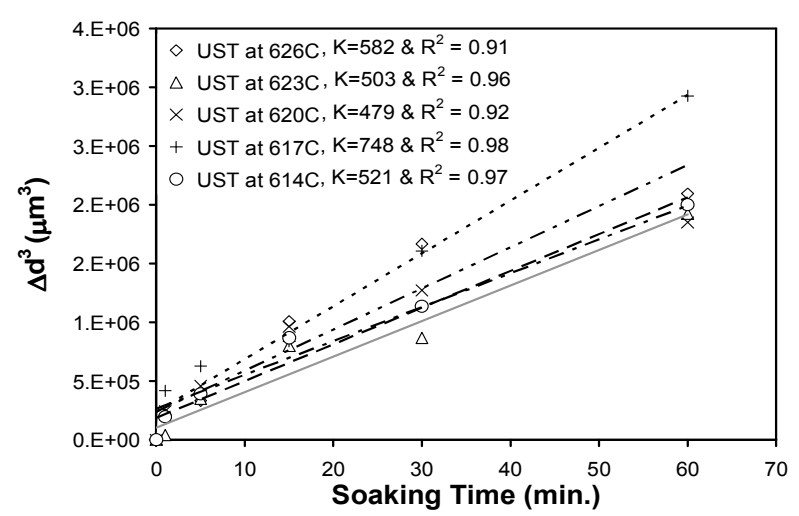

(b)

Fig. 5 Dependence of isothermal coarsening on ultrasonic treatment condition and soaking time: (a) inner zone and (b) outer zones of the ingot.

The grain growth during the current soaking experiments of the UST-treated samples is studied using the LSW (Lifshitz-Slyozov and Wanger) relationship [5,6]:

$$
d_{t}^{3}=d_{0}^{3}+K t
$$

where $d_{t}$ is the globule size after soaking time $t, d_{0}$ is the starting globule size and $K$ is the growth rate constant and is usually expressed as $\mu \mathrm{m}^{3} / \mathrm{s}$. The diagrams of Fig. 5 show the dependence of isothermal coarsening $\left(\Delta \mathrm{d}^{3}=\mathrm{d}_{\mathrm{t}}{ }^{3}-\mathrm{d}_{0}{ }^{3}\right)$ on the ultrasonic treatment temperature and the soaking time at both casting zones. The calculated growth rate constants $\mathrm{K}$ (in $\mu \mathrm{m}^{3} / \mathrm{s}$ ) and the R-squared values for each treatment condition are shown as well in the diagrams.

It can be seen from Fig. 5 that the growth rates are generally higher in the inner regions of the ingot than the outer regions. The growth rate constants are generally lower for the outer zones of the ingot than those of the inner regions of the same UST conditions, namely: $479-748 \mu \mathrm{m}^{3} / \mathrm{s}$ and 534$675 \mu \mathrm{m}^{3} / \mathrm{s}$, respectively. The growth rate of the conventionally cast sample (No UST-M condition) is the highest of all the studied conditions with a K-value of $953 \mu \mathrm{m}^{3} / \mathrm{s}$. It is also worth noting here that the growth rates of the samples produced by UST in the liquid state (i.e., 626, 623 and $620^{\circ} \mathrm{C}$ ) are generally lower than those of the samples treated in the semi-solid temperatures, i.e., 617 and $614^{\circ} \mathrm{C}$. In other words, the samples produced based on refining by nucleation (grain refiner + UST in the liquid) have growth rates less than those produced based on refining by grain refiner and dendrite arm fragmentation (UST in the semisolid range). In this sense, our results are in agreement with Arnberg et al.[9], who reported that the MHD cast ingots were found to have a high fraction of grains having low-angle and twin grain boundaries than the grain refined alloys and thus undergo fast grain growth through simultaneous agglomeration/coalescence and Ostwald ripening.

However, the growth rate constants reported for MHD-cast A356 during soaking at $580^{\circ} \mathrm{C}$ (i.e., $1043-3099 \mu \mathrm{m}^{3} / \mathrm{s}$ for $600-1200 \mathrm{~W}$ MHD stirring power [5]) are much higher than our current values. This large difference cannot be attributed to the effect of Ti-grain refining in our samples since the 
starting materials before the ripening experiments do not show that large difference in globule sizes, i.e., $75-83 \mu \mathrm{m}$ and 59-70 $\mu \mathrm{m}$ in the MHD and UST treated samples, respectively, but rather to the high potential of the MHD-cast materials for grain coalescence. This deduction can be further supported by the grain growth of the non-UST-treated sample which was $97 \mu \mathrm{m}$ in grain size in the as-cast condition and exhibited growth with a rate constant of $953 \mu \mathrm{m}^{3} / \mathrm{s}$, a value being much lower than those of the MHD-cast materials. The UST-treated samples, on the other hand, most likely adopt the slow Ostwald ripening as the main growth mechanism as can be learned from the small globule sizes (about $100 \mu \mathrm{m}$ after $15 \mathrm{~min}$. soaking at $586^{\circ} \mathrm{C}$, Fig.1) and the very low fraction of entrapped eutectic observed, which is an indication of grain agglomeration. The slow growth of the $\alpha$-solid in the UST-treated samples is a very advantageous point which reveals the feasibility and competence of UST as a potential route for thixotropic feedstock production.

\section{Conclusions}

The effect of reheating to the semisolid state (soaking treatment) on the microstructure evolution of the A356 aluminum alloy prepared by ultrasonic melt treatment was studied in this paper. The results showed that in general the longer the soaking process the larger and the more round the grains obtained. Higher roundness occurs at shorter soaking times in the fine-grained as-cast samples, and at longer times in the inhomogeneous or the coarser-grained as-cast structures. The optimum thixotropic condition (high roundness, 0.72 , and small globule sizes $<90 \mu \mathrm{m}$ ) are achieved after $5 \mathrm{~min}$. soaking in the samples treated by UST at 623 and $620^{\circ} \mathrm{C}$, which is the typical soaking time dictated by the industrial practice in SSM. The amount of entrapped eutectic as observed after soaking treatments is uniquely very small, suggesting that the UST- treated ingots will have better formability in the semisolid state. The growth rate constants are substantially low: in the order of $479-748 \mu \mathrm{m}^{3} / \mathrm{s}$. These growth rate constants are much lower than those reported for MHD-cast A356 ingots. The growth rates of the samples produced by UST in the liquid state (i.e., 626,623 and $620^{\circ} \mathrm{C}$. Note that liquidus temperature is $619^{\circ} \mathrm{C}$ ) are lower than those of the samples treated in the semi-solid temperatures, i.e., 617 and $614^{\circ} \mathrm{C}$. The Ostwald ripening is most likely the dominant growth mechanism in the UST-treated samples during the soaking treatments. These results reveal the feasibility and competence of UST as a potential route for thixotropic feedstock production.

\section{References}

[1] Z. Fan: Int. Mater. Rev., vol. 47 (2002), p. 49.

[2] I. Brodova, P. Popel, G. Eskin: Liquid Metal Processing (Taylor \& Francis, London 2002).

[3] T. Meek, X. Jian, H. Xu, Q. Han, report ORNL/TM-2005/125, U.S. Dept. of Energy, 2006.

[4] W. Khalifa, Y. Tsunekawa, M. Okumiya, submitted to the 10th Asian Foundry Congress, 2008.

[5] E. Zoqui, M. Shehata, M. Paes, V. Kao, E. Es-Sadiqi, Mater. Sci. Eng., vol. A325 (2002), p. 38.

[6] E. Tzimas, A. Zavaliangos, Mater. Sci. Eng., vol. A289 (2000), p. 228.

[7] H. Jung, J. Mater. Process. Technol., vol. 105 (2000), p. 176.

[8] V. Franetovic, R. Lints and J. Carter, Aluminum 2003, S.K. Das (edit.), TMS, 2003, p. 133.

[9] L. Arnberg, A. Bardal, H. Sund, Mater. Sci. Eng., vol. A262 (1999), p. 300. 